\title{
Hard X-ray emission of cataclysmic variables observed by INTEGRAL
}

\section{Rudolf Gális ${ }^{*} 1,2,3$, Dominique Eckert ${ }^{2}$, Stéphane Paltani ${ }^{2}$, Filip Münz ${ }^{4}$, Matúš Kocka ${ }^{1}$ and René Hudec ${ }^{1}$}

\author{
${ }^{1}$ Astronomical Institute of the Academy of Sciences of the Czech Republic \\ Fričova 298, 25165 Ondřejov, Czech Republic \\ ${ }^{2}$ ISDC - Data Centre for Astrophysics \\ Chemin d'Écogia 16, CH-1290 Versoix, Switzerland \\ ${ }^{3}$ Faculty of Sciences, University of P. J. Šafárik \\ Moyzesova 16, 04154 Košice, Slovak Republic \\ E-mail: galiseta3.sk \\ ${ }^{4}$ Instituto di astrofisica spaziale e fisica cosmica, INAF \\ via Gobetti 101, 40129 Bologna, Italia
}

\begin{abstract}
Cataclysmic variables and especially intermediate polars represent significant fraction of all INTEGRAL detections in hard X-ray/soft $\gamma$ ray. Our deep survey of all known intermediate polars revealed 5 new sources, previously undetected by INTEGRAL. Our analysis based on all available observational data from INTEGRAL/IBIS and INTEGRAL/JEM-X showed that the fluxes of some intermediate polars are long-term variable. Moreover this hard X-ray/soft $\gamma$ ray variability is correlated with the changes in optical spectral band. The broad-band spectra $(3-100 \mathrm{keV})$ of surveyed intermediate polars can be well fitted by a thermal bremsstrahlung model, with reflection from an optically thick cold medium (the surface of the white dwarf) in some cases.
\end{abstract}

The 7th INTEGRAL Workshop

Copenhagen, Denmark

September 8- 112008

\footnotetext{
${ }^{*}$ Speaker
} 


\section{Introduction}

Cataclysmic variables (CVs) are a special group of eruptive variable stars with more than 1600 particular objects [1]. They manifest strong activity related to mass accretion in the whole spectrum from radio up to $\gamma$ rays on the time-scales from seconds to millions of years.

CVs are close binary systems consisting of a hot white dwarf (WD) and red main sequence star (spectral type $\mathrm{M}$ or $\mathrm{K}$ ), which fills the volume of its inner Roche lobe and transfers matter to the vicinity of the WD [2]. According to strength of WD magnetic field, this matter is creating an accretion disk or follows magnetic lines and falls onto the surface of the WD. Magnetic CVs are a small sub-set of all catalogued CV systems and fall into two categories: polars and intermediate polars (IPs). In IPs, the WD magnetic field $\left(10^{6}-10^{7} \mathrm{G}\right)$ is generally not strong enough to disrupt disc entirely (as in polar systems) and simply truncates the inner part of the disc, resulting in an accretion flow that is channelled down towards the magnetic poles and onto the WD surface.

In a simple model of a column of gas impacting the atmosphere of the WD, a shock will form [3] and hard X-ray/soft $\gamma$-ray emission will result from thermal bremsstrahlung cooling by free electrons in the hot post-shock region (PSR) with $k T \sim(5-60) \mathrm{keV}$ [2]. Reflection of the bremsstrahlung photons at the WD surface also contributes to the hard X-ray spectrum [4]. The role of the two-temperature plasma was studied and the dipole magnetic funnelling was considered in the recent investigations [5]. The influence of Compton scattering on the broadband X-ray spectra of IPs is significant only at high accretion rates and large WD masses [6]. The plasma temperature in the PSR depends on the free-fall velocity at the WD surface and, therefore, the X-ray spectra of IPs can be used to determine the WD mass [] $]$.

The IPs are the most luminous and the hardest X-ray sources among accreting WDs. In hard X-rays, these objects seem to be more luminous (up to the factor of 10) than polars [7]. In the strongly magnetized $\left(B \geq 10^{7} \mathrm{G}\right)$ polar systems, cyclotron radiation is an important cooling mechanism, which suppresses the high temperature bremsstrahlung emission, whilst it should be negligible for the IPs. This could explain why the majority of the CVs observed in the hard X-ray band are IPs.

IPs have been recently proposed to be the dominant X-ray source population detected near the Galactic centre [8]. IPs also contribute significantly to the X-ray diffuse Galactic ridge emission [9]. Moreover, most of the known CVs detected by INTEGRAL are IPs.

\section{Observations and analysis}

We used publicly available observational data from INTEGRAL/IBIS to search for hard X-ray emission of all known IPs. This observational material represents over 8000 individual pointings obtained during almost 5 years (total exposure time over $23 \mathrm{Msec}$ ). The list of the IPs was extracted from the Living Edition of a Catalogue and Atlas of Cataclysmic Variables [1]. In total, we found 65 IPs in this catalogue. The mosaic were prepared in (20-40) $\mathrm{keV}$, as majority of the previously detected IPs emit mainly in this energy band. 
We used all available observations from INTEGRAL/IBIS to study possible variability of V 1223 Sgr in hard X-ray/soft $\gamma$ ray spectral bands. This observational material represents 1375 individual pointings (total exposure time $1405.5 \mathrm{ksec}$ ) obtained during almost three years (MJD 52710.38 - 53809.25). INTEGRAL/OMC data were used to looking for long-term variability of V1223 Sgr in optical spectral band.

We used all available data from INTEGRAL/JEM-X and INTEGRAL/IBIS to study broadband X-ray spectra of the selected IPs: V 709 Cas, GK Per, V 1223 Sgr, IGR J16167-4957 and V2400 Oph. To process the data, we created mosaic images of the corresponding regions to extract the list of all detected sources, and then we extract the spectra.

Observational data used in our analysis were processed by INTEGRAL's Offline Standard Analysis Package OSA7. The libraries grid.py and ms_suite.py developed by FM were also used for the data processing and preparation of the final mosaics. Spectral analysis was performed with the XSPEC version 11.3.2 [10].

\section{Results}

We performed very careful inspection and analysis of all obtained mosaics. In some cases (V1062 Tau, V2306 Cyg) the analysis was complicated by presence of very bright source (Crab, Cyg X-1) in the resulting mosaics. We can conclude that we are not able to find significant detection of the X-ray emission in the mosaics for 43 known IPs. On the other hand, in the 5 cases, namely AO Psc, TV Col, XY Ari, V1062 Tau and V2306 Cyg we can declare new, previously unreported detection of the X-ray emission in the (20-40) $\mathrm{keV}$ mosaics. The corresponding fluxes as well as their errors are listed in Table 1.

Now, the sample of IPs detected in (20-40) keV energy band has 18 members, which represents almost $25 \%$ of all known IPs. Nevertheless, some IPs are not detectable even we have significant exposure time (more than $4 \mathrm{Msec}$ ) for these sources. This fact can be related with activity state of these close interacting binaries. So, in the next investigation we concerned in correlation between the activity state and X-ray emission of the IPs.

Table 1. List of newly detected IPs.

\begin{tabular}{lccccc}
\hline \hline \multicolumn{1}{c}{ Source } & $\begin{array}{c}\text { RA } \\
(\mathrm{J} 2000)\end{array}$ & $\begin{array}{c}\text { Dec } \\
(\mathrm{J} 2000)\end{array}$ & $\begin{array}{c}F_{(20-40) \mathrm{keV}} \\
{\left[10^{-12} \mathrm{erg} \mathrm{cm}^{-2} \mathrm{~s}^{-1}\right]}\end{array}$ & Significance & $\begin{array}{c}\text { Exp. Time } \\
{[\mathrm{ksec}]}\end{array}$ \\
\hline \hline XY Ari & $02: 56: 08.10$ & $+19: 26: 34.0$ & $17.8 \pm 4.7$ & 3.9 & 42.0 \\
V1062 Tau & $05: 02: 27.47$ & $+24: 45: 23.4$ & $11.9 \pm 1.5$ & 7.8 & 462.0 \\
TV Col & $05: 29: 25.52$ & $-32: 49: 04.0$ & $28.4 \pm 1.1$ & 25.9 & 990.0 \\
V2306 Cyg & $19: 58: 14.48$ & $+32: 32: 42.2$ & $7.2 \pm 0.5$ & 12.9 & 1713.0 \\
AO Psc & $22: 55: 17.99$ & $-03: 10: 40.0$ & $16.1 \pm 1.8$ & 8.7 & 273.0 \\
\hline \hline
\end{tabular}




\subsection{Long-term variability of V1223 Sgr}

Intermediate polar V $1223 \mathrm{Sgr}$ is a bright X-ray source (4U 1849-31) with possible X-ray flare activity. Unusual high energy burst (a flare lasting for $\sim 3.5 \mathrm{hrs}$ with a peak flux $\sim 3$ times that of the average) was observed by INTEGRAL/IBIS at MJD $=52743$ [11]. A short-term burst (the single outburst lasted 6 - $24 \mathrm{hrs}$ and resulted in an increase in flux by a factor of 3 ) has also been detected from this system in the optical [12]. These outbursts are probably a result of disk instabilities or an increase in mass transfer from the secondary but there is no correlation between optical and X-rays burst activity. Moreover the episodes of deep low state (decrease by several magnitudes) of V $1223 \mathrm{Sgr}$ in optical band were also detected [13].

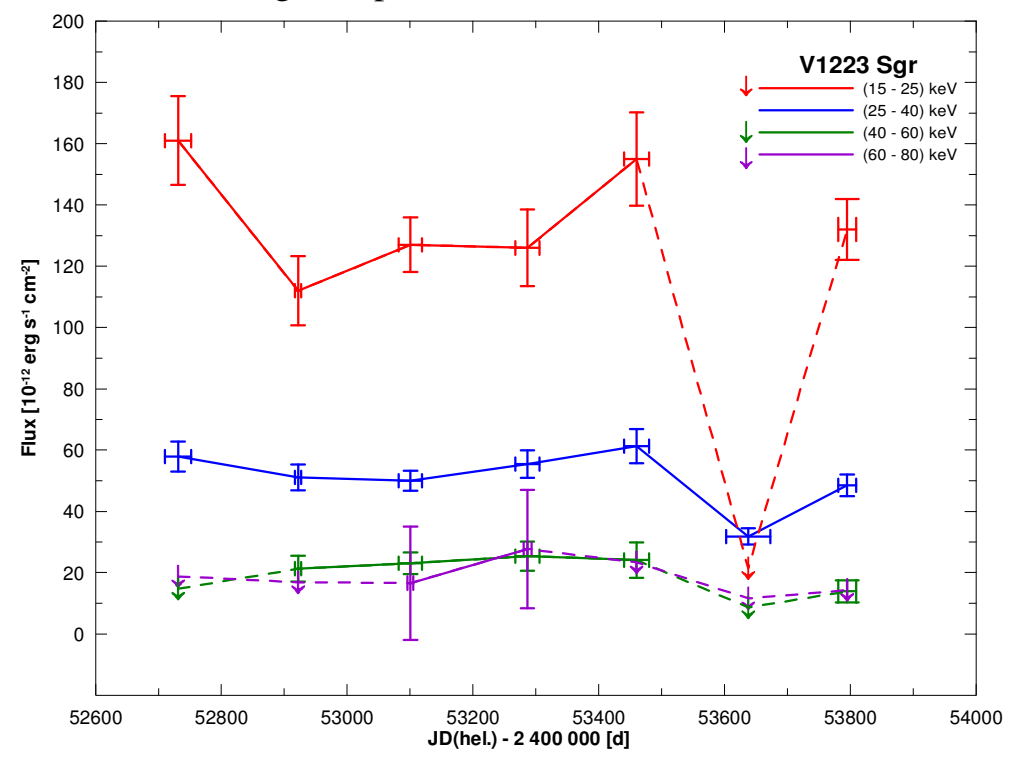

Figure 1. INTEGRAL/IBIS light curves of V $1223 \mathrm{Sgr}$ in corresponding energy bands. The arrows represent $3 \sigma$ upper limits.

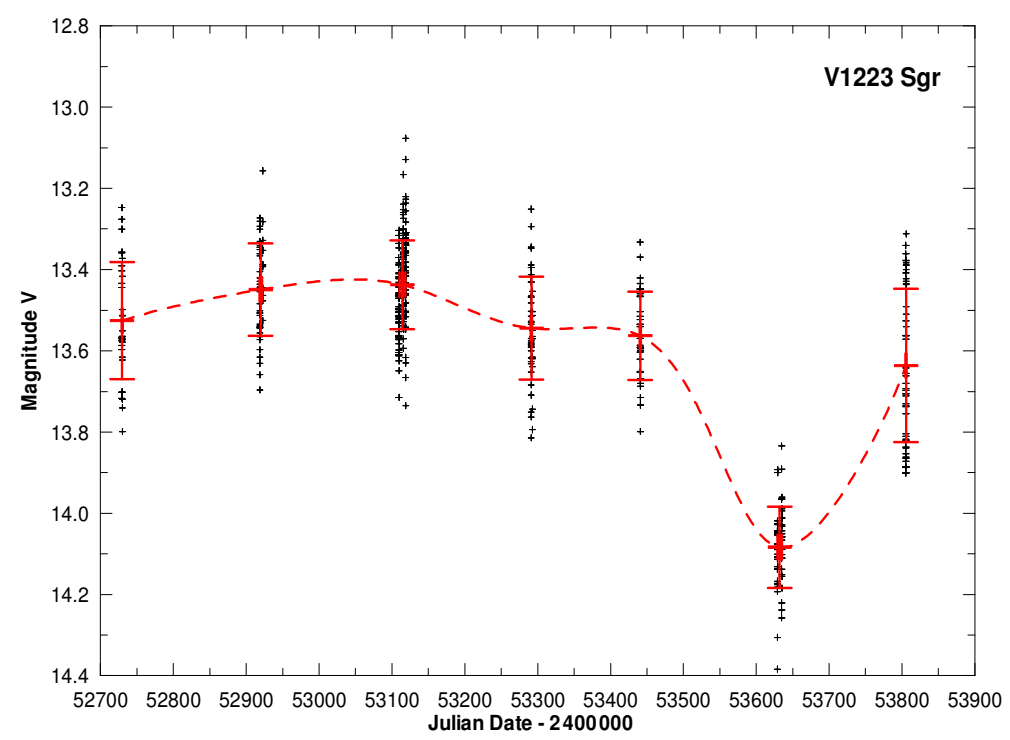

Figure 2. INTEGRAL/OMC light curve of V $1223 \mathrm{Sgr}$ in optical spectral band. The red crosses represent average values of magnitude in the corresponding seasons. 
The overall INTEGRAL/IBIS mosaics showed that V $1223 \mathrm{Sgr}$ is detectable up to $80 \mathrm{keV}$. During monitored period the mean fluxes of this object were $(103.0 \pm 3.9) \times 10^{-12} \mathrm{erg} \mathrm{cm}^{-2} \mathrm{~s}^{-1}$, $(46.4 \pm 1.4) \times 10^{-12} \mathrm{erg} \mathrm{cm}^{-2} \mathrm{~s}^{-1},(15.1 \pm 1.5) \times 10^{-12} \mathrm{erg} \mathrm{cm}^{-2} \mathrm{~s}^{-1}$ and $(12.3 \pm 2.0) \times 10^{-12} \mathrm{erg} \mathrm{cm}^{-2} \mathrm{~s}^{-1}$ in (15 - 25) keV, (25 - 40) keV, (40 - 60) keV and (60 - 80) keV band, respectively.

The inspection of the data showed that observations were obtained during 7 separate seasons. As the next step we splitted the data according these seasons to investigate long-term $\mathrm{X}$-ray $/ \gamma$ ray variability. The light curves are displayed in Figure 1 . It is clear that the fluxes especially in (15 - 25) keV and (25 - 40) keV bands are long-term variable with significant drop around MJD 53650 .

The optical light curve of V1223 Sgr based on available INTEGRAL/OMC data is showed in Figure 2. We can see that optical brightness of this source is long-term variable. Moreover these variations are correlated with the changes in $(15-25) \mathrm{keV},(25-40) \mathrm{keV}$ and (40 - 60) $\mathrm{keV}$ spectral bands with correlation coefficients $0.81,0.82$ and 0.89 , respectively. Significant part of optical emission from IPs is produced by a hot spot, where the matter from donor star interacts with outer rim of the accretion disk. X-ray emission is produced by interaction of the accreting matter with the WD surface. So, the emission in both optical and X-ray bands is related with mass transfer and therefore observed variations are probably caused by the changes in mass accretion rate.

\subsection{Broad-band spectra of IPs}

In all but one case (see below) the broad-band (3-100) $\mathrm{keV}$ spectra of investigated IPs based on all available observation data from INTEGRAL/JEM-X and INTEGRAL/BBIS were well fitted by a thermal bremsstrahlung model with post-shock temperatures $k T \sim(20-25) \mathrm{keV}$. The best fit parameters are listed in Table 2 . The fluxes $F_{(20-40) \mathrm{kev}}$ and $F_{(40-100) \mathrm{keV}}$ were extracted from the mosaic images. The broad-band (3-100) $\mathrm{keV}$ spectra together with the bremsstrahlung model fits are plotted in Figure 3-7.

Table 2. The best fit parameters of the investigated IPs.

\begin{tabular}{lcccc}
\hline \multicolumn{1}{c}{ Source } & $\mathrm{kT}[\mathrm{keV}]$ & $\chi^{2} /$ d.o.f & $\begin{array}{c}F_{(20-40) \mathrm{keV}} \\
{\left[10^{-12} \mathrm{erg} \mathrm{cm}^{-2} \mathrm{~s}^{-1}\right]}\end{array}$ & $\begin{array}{c}F_{(40-100) \mathrm{keV}} \\
{\left[10^{-12} \mathrm{erg} \mathrm{cm}^{-2} \mathrm{~s}^{-1}\right\}}\end{array}$ \\
\hline \hline V709 Cas & $24.4_{-1.4}^{+1.5}$ & $22.6 / 16$ & $27.0 \pm 0.3$ & $18.3 \pm 0.5$ \\
GK Per & $22.5_{-3.4}^{+4.5}$ & $9.9 / 10$ & $17.5 \pm 1.3$ & $<12.2^{*}$ \\
V1223 Sgr & $23.7_{-1.3}^{+1.4}$ & $12.4 / 10$ & $48.1 \pm 0.7$ & $22.1 \pm 1.3$ \\
IGR J16167-4957 & $19.5_{-1.7}^{+2.0}$ & $7.7 / 8$ & $12.4 \pm 0.4$ & $4.9 \pm 0.8$ \\
V2400 Oph & $24.6 \pm 1.6$ & $24.4 / 9$ & $24.6 \pm 0.4$ & $6.9 \pm 0.7$ \\
\hline \hline
\end{tabular}

* We used $3 \sigma$ upper limit.

In the broad-band X-ray spectra of V709 Cas and V1223 Sgr we can see significant excess of emission in the $(6-10) \mathrm{keV}$ band wich can be related to presence of $\mathrm{Fe}$ emission line in the spectra of these objects. Unfortunately, the resolution of our spectra did not allow for a deeper analysis. 


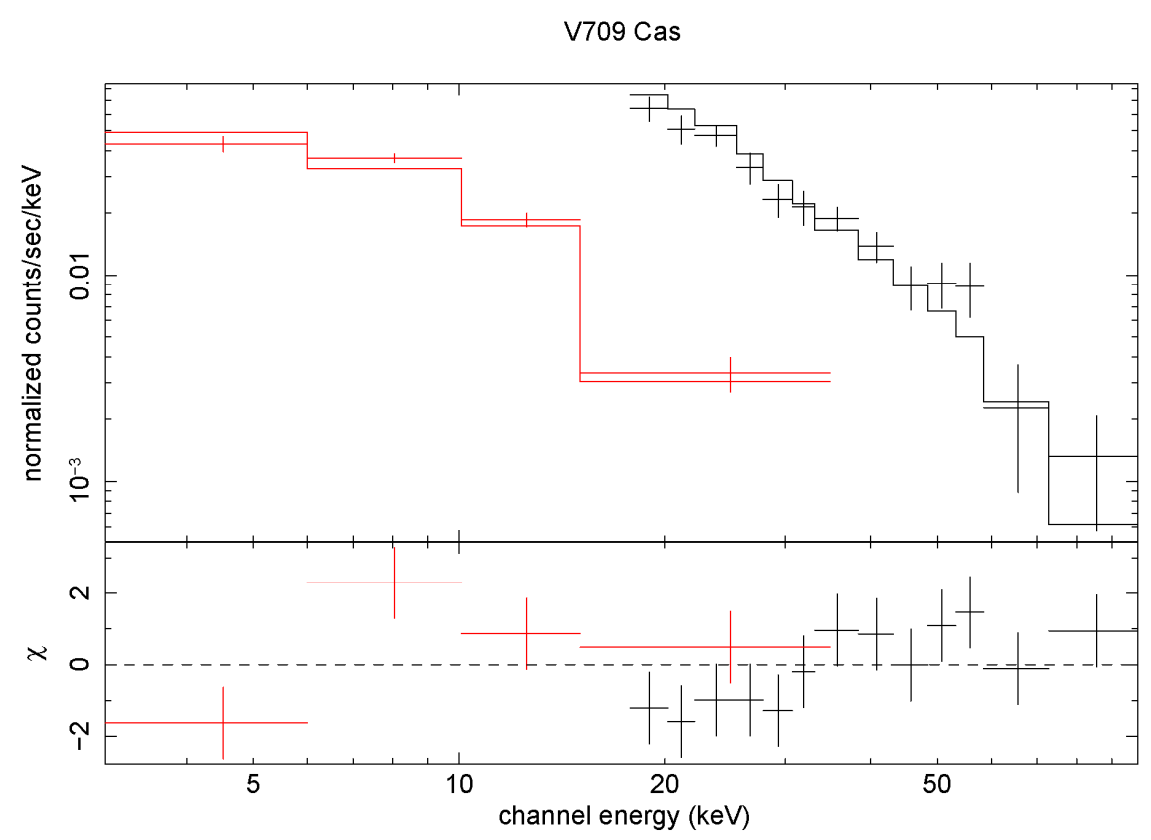

Figure 3. INTEGRAL/JEM-X (red) and INTEGRAL/IBIS (black) broadband spectrum of V709 Cas fitted by a thermal bremsstrahlung model with temperature $24.4_{-1.4}^{+1.5} \mathrm{keV}$.

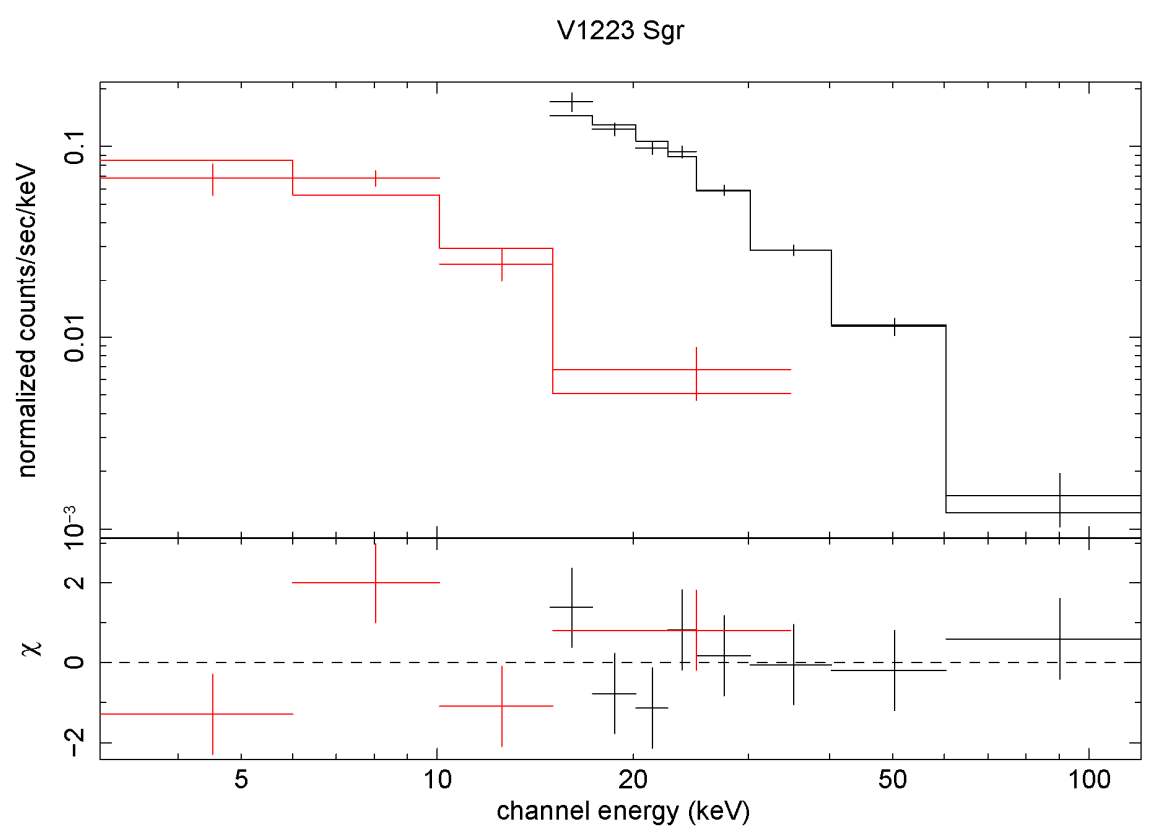

Figure 4. INTEGRAL/JEM-X (red) and INTEGRAL/IBIS (black) broadband spectrum of V1223 Sgr fitted by a thermal bremsstrahlung model with temperature $23.7_{-1.3}^{+1.4} \mathrm{keV}$.

The exccess of emision around $30 \mathrm{keV}$ in the spectrum of GK Per is probably due to reflection, but for a detail analysis it will be necessary to extract a better signal-to-noise spectrum. 


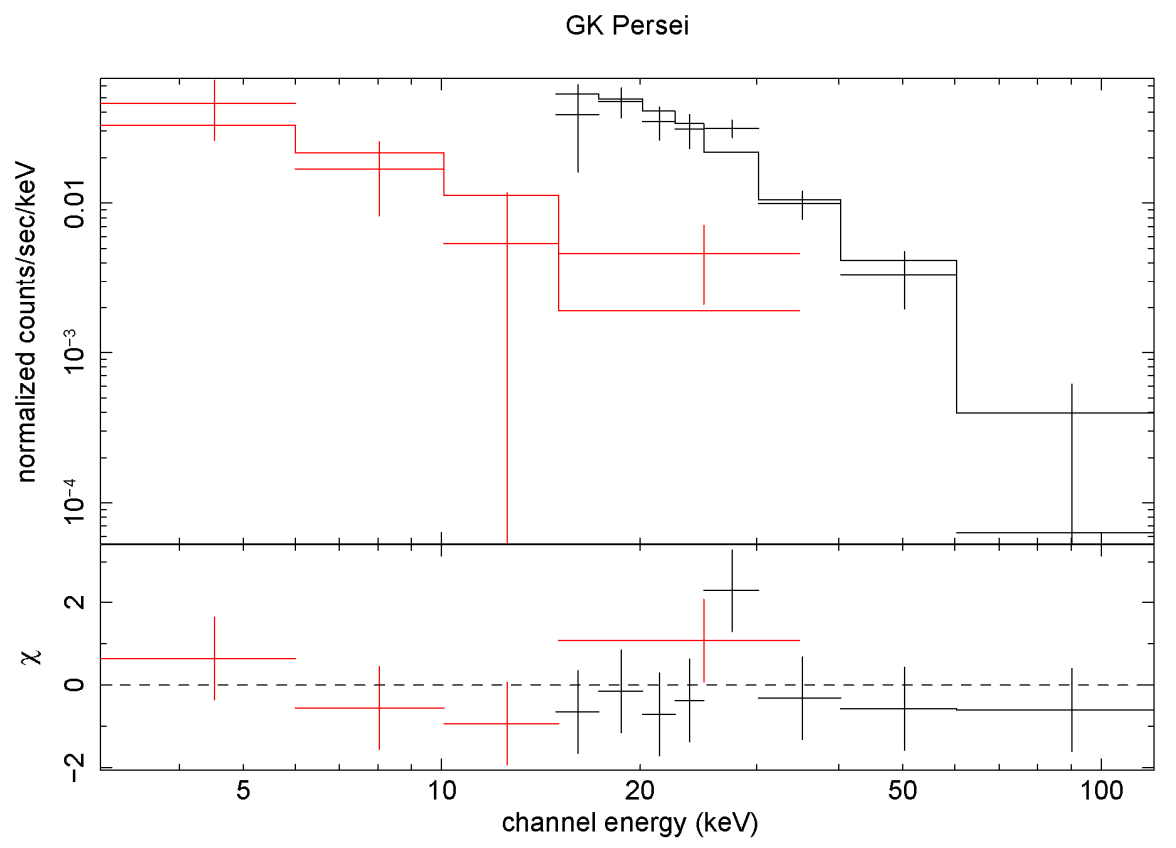

Figure 5. INTEGRAL/JEM-X (red) and INTEGRAL/IBIS (black) broadband spectrum of GK Per fitted by a thermal bremsstrahlung model with temperature $22.5_{-3.4}^{+4.5} \mathrm{keV}$.

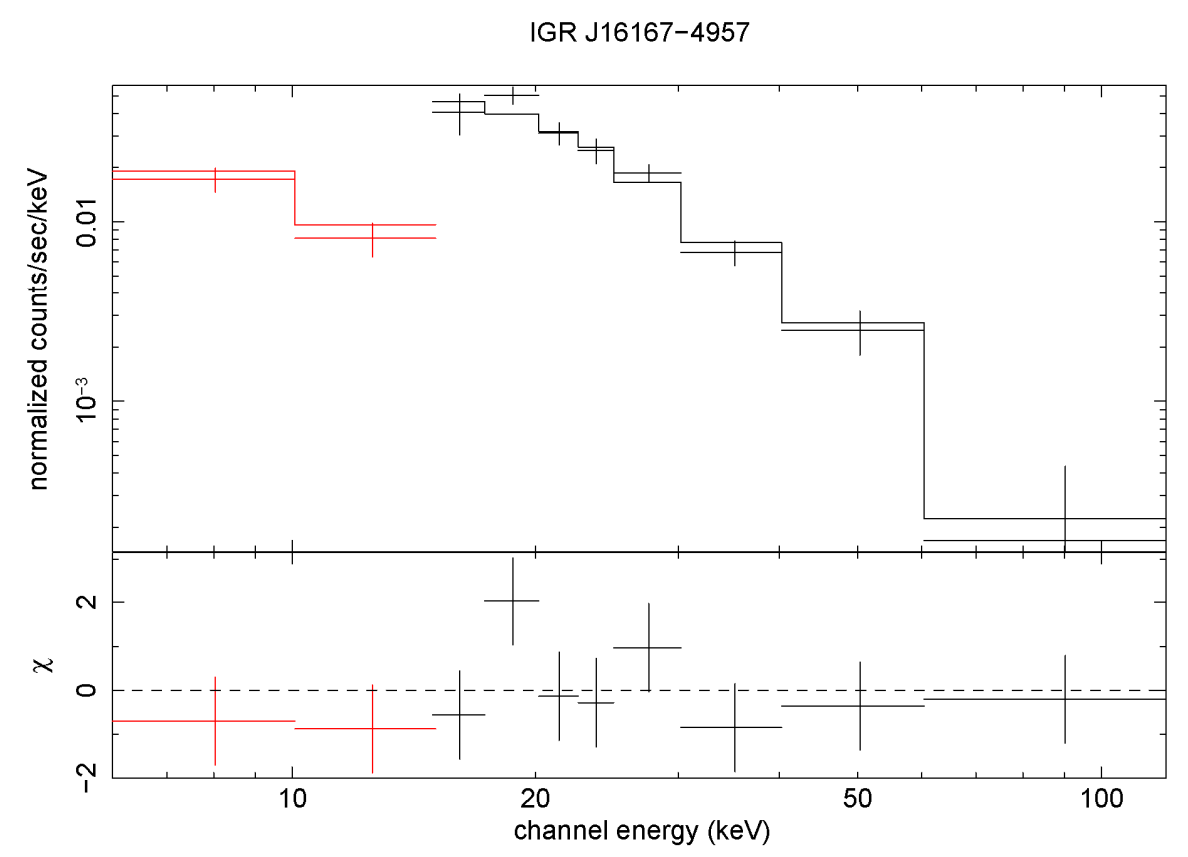

Figure 6. INTEGRAL/JEM-X (red) and INTEGRAL/IBIS (black) broadband spectrum of the source IGR J16167-4957 fitted by a thermal bremsstrahlung model with temperature $19.5_{-1.7}^{+2.0} \mathrm{keV}$. We prepared only two spectral bins for INTEGRAL/JEM-X data to achive better signal-to-noise ratio. 


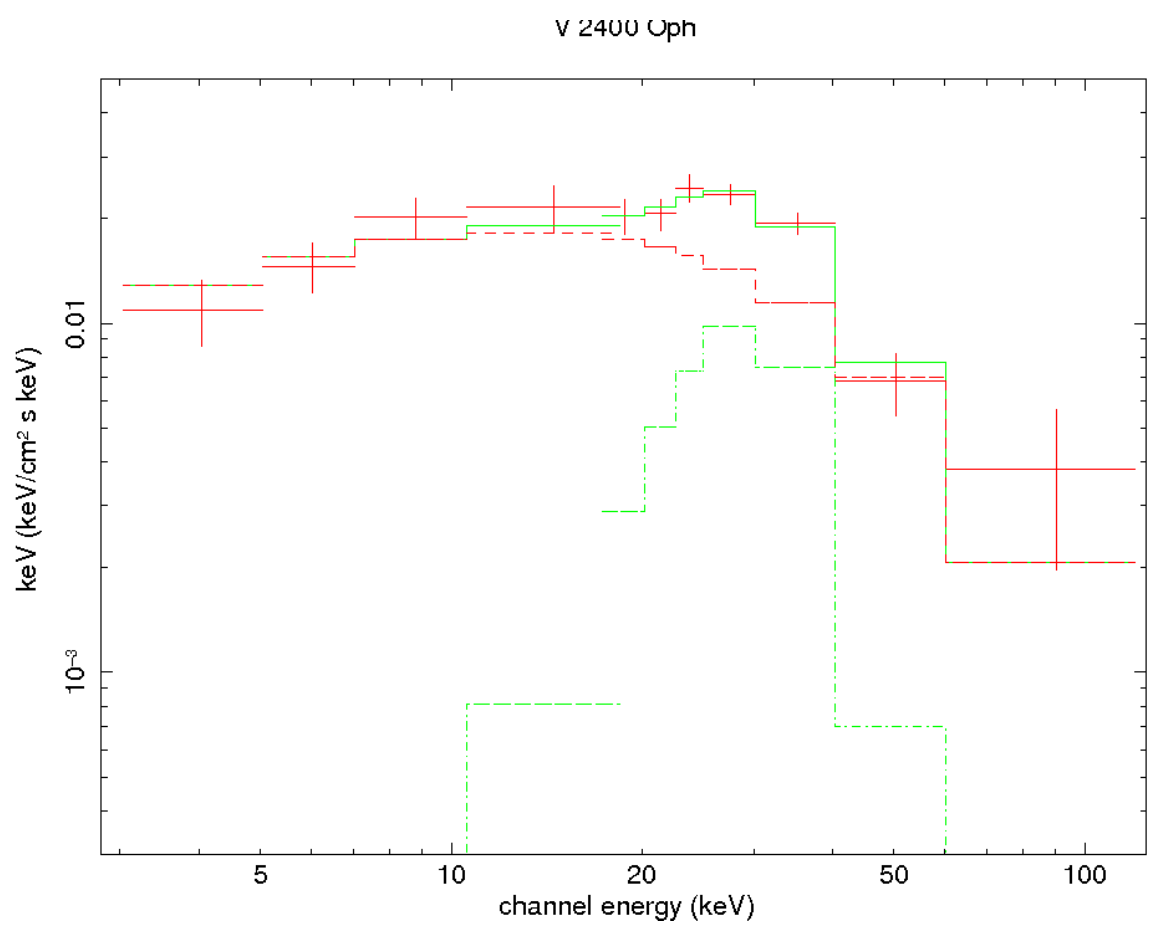

Figure 7. INTEGRAL/JEM-X and INTEGRAL/IBIS broadband spectrum of V2400 Oph fitted by a thermal bremsstrahlung model (temperature $20.3_{-3.1}^{+2.3} \mathrm{keV}$ ) with reflection component at $26 \mathrm{keV}$.

The fit of broad-band X-ray spectrum of V2400 Oph by only a thermal bremsstrahlung model with temperature $k T=(24.6 \pm 1.6) \mathrm{keV}$ is not sufficient to completely describe the data $\left(\chi^{2} /\right.$ d.o.f $\left.=24.4 / 9\right)$. A significant excess $(4.3 \sigma)$ around $\approx 26 \mathrm{keV}$ can be connected with reflection from an optically thick cold medium (the surface of the WD). If we fit this excess by a Gaussian profile (Figure 7), the spectrum of V2400 Oph is very well described with temperature of PSR $k T=20.3_{-3.1}^{+2.3} \mathrm{keV}$ and $\chi^{2} /$ d.o.f $=4.5 / 6$.

\section{Conclusions}

Our deep IP survey based on (20 - 40) keV INTEGRAL/IBIS mosaics revealed the hard $\mathrm{X}$-ray emission of 5 previously undetected IPs. INTEGRAL has already detected $31 \mathrm{CVs}$, mostly IPs (as well as 2 symbiotic systems), which represents a significant fraction of all detections of this mission (5\%). The number of detected CVs will increase in time. Nevertheless some IPs can remain undetectable due to activity state of these close interacting binaries.

Our analysis showed that the fluxes of V1223 Sgr are long-term variable in hard X-ray / soft $\gamma$ ray and this variability is correlated with the changes in optical spectral band. The deep flux drop around MJD 53650 observed in both X-ray and optical bands is probably related to the decrease of the accretion rate in the system.

The broad-band spectra (3-100 keV) of studied IPs can be well fitted by a thermal bremsstrahlung model with the temperature of post-shock region $k T \sim(20-25) \mathrm{keV}$. In the case of V2400 Oph (and possibly GK Per too) there are some evidences for emission excess around $30 \mathrm{keV}$, which can be caused by reflection of X-ray from an optically thick cold medium. 
In conclusion, INTEGRAL proves to be an effective tool to analyse CVs and symbiotic systems in observational window $(3-100) \mathrm{keV}$ so this mission plays important role in the detection as well as in the study of physical processes in these interacting binaries.

\section{Acknowledgement}

The International Gamma-Ray Astrophysics Observatory (INTEGRAL) is an European Space Agency mission with instruments and science data centre funded by ESA member states (especially the PI countries: Denmark, France, Germany, Italy, Spain, Switzerland), Czech Republic and Poland, and with the participation of Russia and the USA. This study was supported by the project ESA PECS INTEGRAL 98023 and the Slovak Academy of Sciences VEGA Grant No. 2/7011/7.

\section{References}

[1] R. A. Downes, R. F. Webbink, M. M. Shara, H. Ritter, U. Kolb, H. W. Duerbeck, A Catalog and Atlas of Cataclysmic Variables: The Living Edition, PASP 113 (2001) 764 [http://archive.stsci.edu/prepds/cvcat/index.html]

[2] B. Warner, Cataclysmic variable stars, Cambridge University Press, Cambridge, 1995

[3] K. Aizu, On the Phase of Pulsar Evolution, Prog. Theor. Phys. 49 (1973) 1184

[4] A. van Teeseling, J. S. Kaastra, J. Heise, Iron K fluorescence and Compton reflection in magnetic cataclysmic variables, A\&A 312 (1996) 186

[5] C. J. Saxton, K. Wu, J. B. G. Canalle, M. Cropper, G. Ramsay, X-ray emissions from twotemperature accretion flows within a dipole magnetic funnel, MNRAS 379 (2007) 779

[6] V. Suleimanov, J. Poutanen, M. Falanga, K. Werner, Influence of Compton scattering on the broadband X-ray spectra of intermediate polars, A\&A (2008) [arXiv:0805.0427]

[7] G. Chanmugam, A. Ray, K. P. Singh, The formation and detection of strongly magnetic white dwarf binaries in globular clusters, ApJ 375 (1991) 600

[8] A. J. Ruiter, K. Belczynski, T. E. Harrison, The Nature of the Faint Chandra X-Ray Sources in the Galactic Center, ApJ 640 (2006) L167

[9] M. Revnivtsev, S. Sazonov, M. Gilfanov, E. Churazov, R. Sunyaev, Origin of the Galactic ridge $X$-ray emission, A\&A 452 (2006) 169

[10] K. A. Arnaud, XSPEC: The First Ten Years, in G. Jacoby, J. Barnes (eds.), Astronomical Data Analysis Software and Systems V, ASP Conf. Ser. 101, Astron. Soc. Pac., San Francisco (1996) 10

[11] E. J. Barlow, C. Knigge, A. J. Bird, A. Dean, D. J. Clark, A. B. Hill, M. Molina, V. Sguera, 20-100 keV properties of CVs detected in the INTEGRAL/IBIS survey MNRAS 372 (2006) 224

[12] S. van Amerongen, J. van Paradijs, Detection of a brief outburst from the intermediate polar V 1223 Sgr, A\&A 219 (1989) 195

[13] P. Garnavich, P. Szkody, Observed low states in DQ Herculis systems, PASP 100 (1988) 1522 